\title{
Quantum-measurement backaction from a Bose-Einstein condensate coupled to a mechanical oscillator
}

\author{
S. K. Steinke, ${ }^{1}$ S. Singh, ${ }^{1}$ M. E. Tasgin, ${ }^{1}$ P. Meystre, ${ }^{1}$ K. C. Schwab, ${ }^{2}$ and M. Vengalattore ${ }^{3}$ \\ ${ }^{1}$ B2 Institute, Department of Physics and College of Optical Sciences The University of Arizona, Tucson, Arizona 85721, USA \\ ${ }^{2}$ Applied Physics, California Institute of Technology, MC 128-95, Pasadena, California 91125, USA \\ ${ }^{3}$ Laboratory of Atomic and Solid State Physics, Cornell University, Ithaca, New York 14853, USA
}

(Received 9 May 2011; published 25 August 2011)

\begin{abstract}
We study theoretically the dynamics of a hybrid optomechanical system consisting of a macroscopic mechanical membrane magnetically coupled to a spinor Bose-Einstein condensate via a nanomagnet attached at the membrane center. We demonstrate that this coupling permits us to monitor indirectly the center-of-mass position of the membrane via measurements of the spin of the condensed atoms. These measurements normally induce a significant backaction on the membrane motion, which we quantify for the cases of thermal and coherent initial states of the membrane. We discuss the possibility of measuring this quantum backaction via repeated measurements. We also investigate the potential to generate nonclassical states of the membrane, in particular Schrödinger-cat states, via such repeated measurements.
\end{abstract}

DOI: 10.1103/PhysRevA.84.023841 PACS number(s): 42.50.Lc, 42.50.Wk, 37.30.+i, 07.10.Cm

\section{INTRODUCTION}

While the foundations of quantum measurement theory were already laid down in the early days of quantum mechanics, quantitative studies are a relatively recent development $[1,2]$. These studies are now driving significant advances in quantum information science and quantum metrology, with major progress initiated by the desire to test Bell's inequalities on the one hand, and by the quest for gravitational wave detection on the other $[3,4]$. A common aspect of these and related studies is the need to quantify, control, and possibly exploit the quantum backaction of one or a series of measurements on a quantum mechanical system.

An important development in this context is the rapid progress witnessed by cavity optomechanics, which makes it increasingly realistic to consider the use of mechanical systems operating in the quantum regime to make precise and accurate measurements of feeble forces and fields [2]. In many cases, these measurements amount to the detection of exceedingly small displacements. In this context, hybrid systems consisting of coupled atomic (or molecular) and nanomechanical systems may prove particularly useful. The robust and scalable infrastructure provided by micro- and nanoelectromechanical systems, coupled with the high-precision-measurement capability of quantum gases [5-8], makes them an attractive combination for sensitive force measurements, as well as for a quantitative study of dissipation and decoherence processes at the quantum-classical interface. As a result, there are ongoing experimental [9,10] and theoretical [11-15] efforts toward coupling mechanical systems to atomic ensembles.

The system that we consider in this paper consists of a mechanical membrane magnetically coupled to a spinor Bose-Einstein condensate (BEC); an arrangement somewhat similar to a system previously considered in Refs. [11,14]. The magnetic coupling, generated via a nanomagnet anchored on the membrane, entangles the membrane to the spin state of the BEC. We may then make an indirect observation of the center-of-mass position of the membrane through a measurement of the spins of the condensed atoms. While such magnetic coupling and concomitant entanglement is possible between the membrane and a thermal gas of spinor atoms (or even a single atom), the BEC has two advantages over the alternatives when used as a position sensor: bosonic stimulation between the atoms that leads to increased signal strength and the suppression of two-body collisions, which gives rise to a much longer phase coherence time when compared to thermalized atoms. Now, the indirect position measurement can, generally speaking, be achieved in two ways: through a strong, or projective, measurement of the spin state of the BEC or through a weak, or dispersive, measurement of the spins. An example of the former would be through a Stern-Gerlach-type measurement. Here, the atoms are subjected to a spatially inhomogeneous field separating out the different spin components. The different spin populations can then be measured via a standard absorptive imaging technique. A likely candidate for the latter type of measurement would be observation of the Larmor frequency through a phase contrast imaging technique, as described in Ref. [5]. Since the Larmor frequency is proportional to the local magnetic field, which is modulated by the motion of the magnetic domain attached to the oscillating membrane, it provides a measure of the membrane motion. Either type of measurement of the BEC spin can induce a backaction on the membrane, modifying its position and/or momentum in proportion to the strength of the measurement. The main goal of this project is thus to quantify the effect of using the BEC as a position sensor on the membrane and to evaluate the feasibility of measuring the backaction of such a measurement. In the case where the backaction is small, we can indeed use the BEC to make ultraprecise measurements of the membrane and, when it is large, it may provide a means to observe the effects of a quantum measurement on a macroscopic object. So far such a quantum effect has only been observed in dilute and isolated systems such as ultracold gases [16].

Due to the scope of this endeavor, we find it convenient to divide our results into three papers. In this, the first paper, we present the experimental setup and develop a simplified 
Hamiltonian to describe the interaction of the membrane and condensate. We derive a general formulation of the system state after a single (or multiple) arbitrary measurement(s) of the condensate's spin state and then present results for specific cases where the measurements are projective. We show that, in principle, the membrane can be put into a nonclassical state by such a procedure. In the second paper we extend these considerations to weak measurements, focusing on quantifying the backaction of indirect observation of the Larmor frequency via phase contrast imaging. In the third paper we will exploit the backaction of repeated measurements and coherent control to achieve quantum control of the state of the membrane, allowing, in principle, the production of squeezed or Schrödinger-cat states. Because the effects of environmental coupling (i.e., decoherence and dissipation) are critical to coherent control protocols, we will also include these effects in the computations of the third paper.

This paper is organized as follows: Section II introduces the system under consideration and derives a model Hamiltonian that describes the magnetic coupling of the membrane to the condensate. Section III discusses the indirect measurement of the membrane center-of-mass position via a strong measurement of the spin of the condensate, assuming that the membrane is initially in a thermal state. In particular, we present the postmeasurement Wigner function of the membrane and interpret its main features in terms of a backaction parameter. Section IV then turns to the case of repeated measurements on this system, illustrating how a sequence of measurements provides a direct signature of the backaction. Section V discusses the possibility of producing highly nonclassical states of the membrane, considering specifically the case where it is initially in a coherent state. We show that repeated measurements typically leave the membrane in a nonclassical state characterized by a nonpositive Wigner distribution. Finally, Sec. VI is a summary and outlook. Some technical details of the calculations and experiment are included as Appendixes.

\section{MODEL}

As mentioned in the introduction, the system under consideration consists of a micromechanical membrane of fundamental-mode frequency $\omega_{m}$ and effective mass $m$, whose center of mass is oscillating, perhaps under the influence of a weak force. Our goal is to develop and analyze a quantum measurement scheme that permits us to characterize the center-of-mass motion of that membrane.

The scheme that we envisage involves integrating this membrane into a hybrid system whose other component is an elongated spinor Bose-Einstein condensate with long axis $z$ (see Fig. 1). The condensate is subjected to a static magnetic field $B_{0}$ along the quantization axis $z$. The membrane is magnetically coupled to the condensate via a magnetic domain anchored at the center of the membrane.

We assume for simplicity that the magnetic domain is a point dipole located at the origin and polarized along the $z$ axis: $\boldsymbol{\mu}_{m}=\mu_{m} \hat{\mathbf{z}}$. The magnetic field due to that dipole is

$$
\mathbf{B}(\mathbf{r})=\frac{\mu_{0}}{4 \pi} \frac{1}{r^{3}}\left[3\left(\boldsymbol{\mu}_{\boldsymbol{m}} \cdot \hat{\mathbf{r}}\right) \hat{\mathbf{r}}-\boldsymbol{\mu}_{m}\right] .
$$
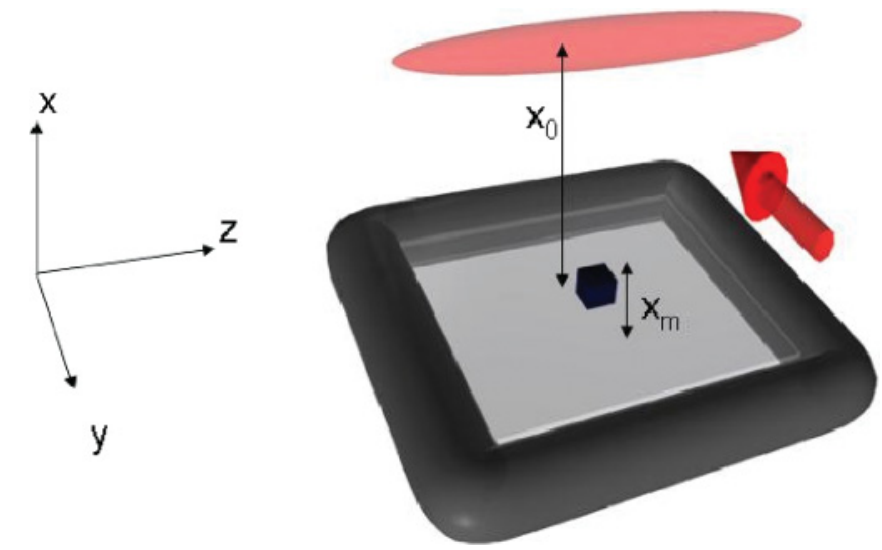

FIG. 1. (Color online) Proposed experimental setup for backaction detection, involving a magnetic particle located at the center of a vibrating membrane and polarized along the $z$ axis. This setup produces a spatially inhomogeneous magnetic field that is detected by the BEC. The BEC's long axis is along the $z$ direction, the probe light (red arrow) is along $y$, and the membrane oscillations are along the $x$ axis.

The small spatial variation of the resulting magnetic field along the long axis of the condensate results in a variation of its Larmor precession frequency. As we show in the following, this dependence permits us to characterize the expectation value of the center-of-mass mode of oscillation of the membrane.

The Zeeman interaction between a single atom in the condensate and the total magnetic field $\mathbf{B}_{0}+\mathbf{B}(\mathbf{r})$ is described by the interaction Hamiltonian

$$
\begin{aligned}
V & =-\boldsymbol{\mu}_{a} \cdot\left[\mathbf{B}_{0}+\mathbf{B}(\mathbf{r})\right] \\
& =\mu_{B} g_{F}\left[F_{x} B_{x}+F_{y} B_{y}+F_{z}\left(B_{0}+B_{z}\right)\right],
\end{aligned}
$$

where $F_{i}$ is the $i$ th component of the spin-1 operator, $\mu_{B}$ is the Bohr magneton, and $g_{F}=2$ for alkali-metal atoms. Because of the extremely low temperature of the condensate and the relatively small trapping frequencies employed (on the order of 10-100 Hz), we will neglect the kinetic energy of the atoms and the trapping potential in our model. As a result of the spatial dependence of the magnetic field, atoms at different positions along the long axis of the condensate precess at different frequencies and dephase over a period of time. It is this $z$-dependent phase difference that is picked up in spin-population measurements.

We note that the transverse dependence of the magnetic field also results in an inhomogeneous broadening of the Larmor frequency. This broadening can be minimized by elongating the condensate along its $z$ axis and by confining it more tightly in the transverse directions. In the following we consider for simplicity a condensate that is almost one dimensional and confined to a region close to $y=0$, so that $B_{y} \approx 0$ and $F_{y} B_{y} \approx 0$. Furthermore, close to $z=0$ (i.e., for the fraction of the BEC directly above the dipole) the magnetic field $\mathbf{B}(\mathbf{r})$ is predominantly in the $z$ direction provided that $x_{0}$, which is the equilibrium distance between the BEC and the membrane, is much greater than the relevant coordinates $y$ and $z$. In that case we can ignore the effects of $B_{x}$ and $B_{y}$ altogether. As shown in detail in Appendix A, for small displacements $x_{m}$ of the 
membrane compared to $x_{0}$, the single-atom magnetic-coupling Hamiltonian (2) reduces to

$$
V=\mu_{B} g_{F} F_{z}\left[B_{0}+\frac{\mu_{0} \mu_{m}}{4 \pi x_{0}^{4}}\left(-x_{0}+3 x_{m}\right)\right] \text {. }
$$

The first and second terms of this expression are independent of time, while the third term, proportional to $x_{m}$, varies sinusoidally in time. We exploit this property by rewriting the magnetic Hamiltonian as

$$
V=\mu_{B} g_{F} F_{z}\left(B_{c}+B_{v}^{\prime} x_{m}\right),
$$

where

$$
\begin{gathered}
B_{c}=B_{0}-\frac{\mu_{o} \mu_{m}}{4 \pi x_{0}^{3}}, \\
B_{v}^{\prime}=\frac{3 \mu_{o} \mu_{m}}{4 \pi x_{0}^{4}} .
\end{gathered}
$$

By treating the condensate as $N$ noninteracting spin-1 atoms under the mean-field approximation and by assuming that the magnetic field acting on the atoms in the detector region is roughly constant, we can easily arrive at the manyatom magnetic-interaction Hamiltonian. It is simply

$$
\begin{aligned}
V_{\mathrm{BEC}} & =\int d^{3} \vec{x} \Psi^{\dagger}(\vec{x}) V \Psi(\vec{x}) \\
& =N \mu_{B} g_{F} F_{z}\left(B_{c}+B_{v}^{\prime} x_{m}\right),
\end{aligned}
$$

where $\Psi(\vec{x})$ is the annihilation operator for an atom at position $\vec{x}$ (i.e., it is a Schrödinger field operator). The total system Hamiltonian of the hybrid BEC-membrane system is then

$$
H=H_{m}+V_{\mathrm{BEC}} \text {. }
$$

Here.

$$
H_{m}=p^{2} /(2 m)+m \omega_{m}^{2} x^{2} / 2
$$

is the membrane Hamiltonian, and we have dropped (and will continue to drop) the subscript in the membrane displacement for compactness (i.e., $x_{m} \rightarrow x$ ). By explicitly combining both terms of $H$ and completing the square for $x$, we express the total system Hamiltonian as

$$
H=\frac{p^{2}}{2 m}+\frac{1}{2} m \omega_{m}^{2}\left(x+A F_{z}\right)^{2}+\left\{\hbar N \Omega_{L 0} F_{z}-\hbar \delta \Omega F_{z}^{2}\right\},
$$

where

$$
A=\mu_{B} g_{F} N B_{v}^{\prime} /\left(m \omega^{2}\right),
$$

which we call the backaction parameter in anticipation of the following sections,

$$
\Omega_{L 0}=\mu_{B} g_{F} B_{c} / \hbar,
$$

which is simply the Larmor precession frequency of the atomic spins, and

$$
\delta \Omega=m \omega_{m}^{2} A^{2} /(2 \hbar) .
$$

\section{SPIN MEASUREMENT}

In this and the following sections, we evaluate the backaction on the membrane of an arbitrary-measurement operator that acts on the spin degree of freedom of the BEC. We consider the cases of a single and multiple spin measurements for a membrane initially prepared either in a thermal or in a coherent state. The analytical expressions provided for the postmeasurement density matrix (and subsequently the Wigner function) are for a general-measurement operator. However, to illustrate the backaction effects in an intuitive manner, we consider in this paper the particular case of simple projective measurements of $F_{y}$, corresponding to all atoms being in the $F_{y}=0,+1$, or -1 state. While this is not the most likely outcome of a typical Stern-Gerlach measurement, it does serve as a simple demonstration of backaction-induced dynamics.

The measurement proceeds in the following way: at time $t=0$, a $\pi / 2$ pulse is applied to the condensate, preparing all atoms in $\left|F_{x}=1\right\rangle$ state. Following this preparation stage, the atomic spins precess about the $z$ axis until a later time $t_{1}$ when the spin-population measurement is made. Additional measurements can be performed at later time intervals $t_{i}, i=$ $2,3, \ldots$ Further precise details of the experimental setup are presented in Appendix D.

Equation (8) already gives a clear indication of the backaction of the measuring apparatus - the condensate-on the membrane. As a result of their coupling, the membrane Hamiltonian is modified from being a harmonic oscillator centered at the origin to one that is shifted by the quantity $A F_{z}$, indicating that the backaction depends on the outcome of a specific spin measurement. Here, we give an explicit description of the measurement process by evaluating the preand postmeasurement density operator of the membrane and the corresponding Wigner function.

\section{A. Density operator}

We assume that the membrane and the condensate are initially uncorrelated,

$$
\rho=\rho_{m}(0) \otimes \rho_{\mathrm{BEC}}(0),
$$

and we denote the initial density matrix elements of the BEC as $\rho_{\alpha, \beta}^{0}$, where $\rho_{\alpha, \beta}=\left\langle\alpha\left|\rho_{\mathrm{BEC}}\right| \beta\right\rangle$ and $\alpha$ and $\beta$ are the various spin states; $\alpha, \beta=\{0, \pm 1\}$. We also assume for now that the membrane center of mass is initially in thermal equilibrium with temperature $T$. Defining

$$
\eta=\hbar \omega_{m} /\left(2 k_{B} T\right),
$$

its density matrix elements in position space are then

$$
\begin{aligned}
\rho_{m}\left(x_{f}, x_{i}, t=0\right)= & \left\langle x_{f}\left|\rho_{m}\right| x_{i}\right\rangle \\
= & \sqrt{\frac{m \omega_{m}}{\pi \hbar} \tanh \eta \exp \left\{-\frac{m \omega_{m}}{4 \hbar}\left[\left(x_{f}+x_{i}\right)^{2}\right.\right.} \\
& \left.\left.\times \tanh \eta+\left(x_{f}-x_{i}\right)^{2} \operatorname{coth} \eta\right]\right\}
\end{aligned}
$$

For $t>0$ the spin components of the condensate undergo a Larmor precession about the $z$ axis. Since $\left[H_{\mathrm{BEC}}, H_{m}+V\right]=$ 0 , we can use the Baker-Hausdorff relation to re-express the 
propagator $U(t)=\exp (-i H t / \hbar)$ as

$$
U(t)=e^{-i t H_{\mathrm{BEC}} / \hbar} e^{-i t\left(H_{m}+V\right) / \hbar} .
$$

This allows us to find the evolution of the system density matrix in a straightforward way. After an interaction time $t_{1}$, this evolution results in the matrix elements of the density operator of the membrane plus condensate system to become

$$
\begin{aligned}
\left\langle\alpha, x_{f}|\rho(t)| \beta, x_{i}\right\rangle= & \rho_{\alpha \beta}^{0} \exp \left[-i \Omega_{L 0}(\alpha-\beta) t_{1}+i \delta \Omega_{L}\left(\alpha^{2}-\beta^{2}\right) t_{1}\right] \sqrt{\frac{m \omega_{m}}{\pi \hbar} \tanh \eta} \exp \left[-\frac{m \omega_{m}}{4 \hbar}\left\{\left[x_{f}+x_{i}\right.\right.\right. \\
& \left.+(\alpha+\beta) A\left(1-\cos \omega_{m} t_{1}\right)\right]^{2} \tanh \eta+\left[x_{f}-x_{i}+(\alpha-\beta) A\left(1-\cos \omega_{m} t_{1}\right)\right]^{2} \operatorname{coth} \eta \\
& \left.\left.+4 i A \sin \omega_{m} t_{1}\left(\alpha x_{f}-\beta x_{i}\right)+2 i A^{2}\left(\alpha^{2}-\beta^{2}\right) \sin \omega_{m} t_{1}\left(2-\cos \omega_{m} t_{1}\right)\right\}\right]
\end{aligned}
$$

That is, the interaction of the membrane with the BEC displaces its center-of-mass motion in both position and momentum by amounts that depend explicitly on the spin components $\alpha$ and $\beta$, as well as on the backaction parameter $A$.

\section{B. Single measurement}

As already discussed, a measurement of arbitrary type, but dependent on $F_{y}$, is carried out on the BEC at time $t_{1}$. The postmeasurement density matrix of the membrane depends on the measurement outcome and is given by

$$
\left\langle x_{f}\left|\rho_{m}\right| x_{i}\right\rangle_{\phi}=\frac{1}{P(\phi)} \operatorname{tr}_{\mathrm{BEC}}\left(W^{\phi} \rho_{\mathrm{sys}}\right) .
$$

Here $\phi$ is the outcome of the $F_{y}$ measurement, $P(\phi)$ is the probability of that outcome, $W^{\phi}$ is the Kraus operator corresponding to the effects of the measurement on the BEC's quantum state, and $\rho_{\text {sys }}$ is the complete system density matrix given in (13). Although Eq. (14) is true for arbitrary measurements (i.e., for arbitrary Kraus representations), in later plots and numerics we take the operators $W^{\phi}$ to be projectors onto the eigenstates of $F_{y}$; namely, the 3 operators $\left|F_{y}=\gamma\right\rangle\left\langle F_{y}=\gamma\right|, \gamma=\{0, \pm 1\}$. This simplified situation displays the salient features of measurement backaction without requiring extensive computations. Such a scenario could be realized through, for example, a Stern-Gerlach apparatus. As mentioned above, we will return to the precise computation of operators $W^{\phi}$ corresponding to the planned experimental setup (Appendix D) in subsequent work.

\section{Phase space representation}

The effect of the measurement on the state of the membrane can be visualized particularly clearly in terms of its Wigner distribution function

$$
W(x, p)=\frac{1}{2 \pi \hbar} \int d \xi e^{-i p \xi / \hbar}\langle x+\xi / 2|\rho| x-\xi / 2\rangle .
$$

For a harmonic oscillator in a thermal state, we have

$$
\begin{aligned}
& W(x, p, t=0) \\
& \quad=\frac{1}{\pi \hbar} \tanh \eta \exp \left\{-\frac{m \omega_{m}}{\hbar}\left[x^{2}+\left(\frac{p}{m \omega_{m}}\right)^{2}\right] \tanh \eta\right\} ;
\end{aligned}
$$

an expression that should be contrasted to the postmeasurement Wigner function, which is found to be

$$
\begin{aligned}
W\left(x, p, t_{1}\right)= & \frac{1}{\pi \hbar} \tanh \eta \frac{1}{P(\phi)} \sum_{\alpha, \beta} W_{\beta \alpha}^{\phi} \rho_{\alpha \beta}^{0} \exp \left[-i \Omega_{L 0}(\alpha-\beta) t_{1}+i \delta \Omega_{L}\left(\alpha^{2}-\beta^{2}\right) t_{1}\right] \\
& \times \exp \left\{-\frac{m \omega_{m}}{\hbar}\left[\left(\left\{x+\frac{A}{2}(\alpha+\beta)\left[1-\cos \omega_{m} t_{1}\right]\right\}^{2}+\left\{\frac{p}{m \omega_{m}}+\frac{A}{2}(\alpha+\beta) \sin \omega_{m} t_{1}\right\}^{2}\right) \tanh \eta\right.\right. \\
& \left.\left.+i A(\alpha-\beta)\left\{x \sin \omega_{m} t_{1}-\frac{p}{m \omega_{m}}\left[1-\cos \omega_{m} t_{1}\right]+\frac{A}{2}(\alpha+\beta) \sin \omega_{m} t_{1}\right\}\right]\right\}
\end{aligned}
$$

At time $t_{1}$, the first line of Eq. (17) simply describes the imposition, due to the evolution of the BEC, of a phase on each term of $W(x, p)$ which is dependent on the measurement outcome and spin indices. More interesting are the last two lines in that expression: The second line describes a term-by-term shift in the initial Gaussian probability distribution. The new phase-space center of each term in the Wigner function depends on the spin indices of that term, and it rotates through phase space at the membrane frequency.
Because the third line in $W\left(x, p, t_{1}\right)$ is imaginary, it results in interference between the terms of the Wigner function (visible below in Fig. 2). From inspection of Eq. (17), we see that the interference will increase with larger $A$. The physical significance of this interference can be understood from the fact that the initial state of the BEC, $\left|F_{x}=1\right\rangle$, is not an eigenstate of the interaction Hamiltonian (proportional to $F_{z}$ ). Thus, one can think of the condensate as experiencing three interaction Hamiltonians simultaneously, one for each of its spin components, and it is the interference between them that 


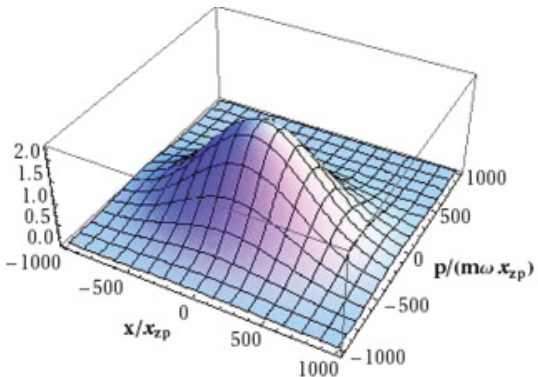

(a) $A=0.01 A_{0}$

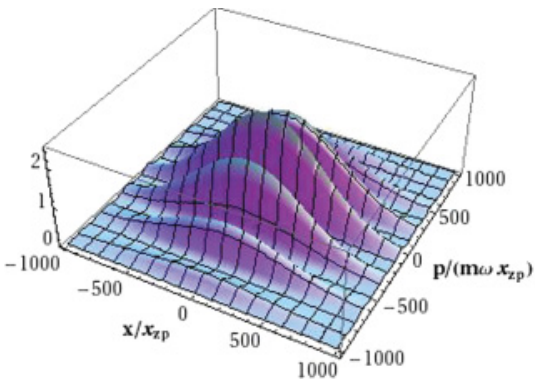

(b) $A=0.1 A_{0}$

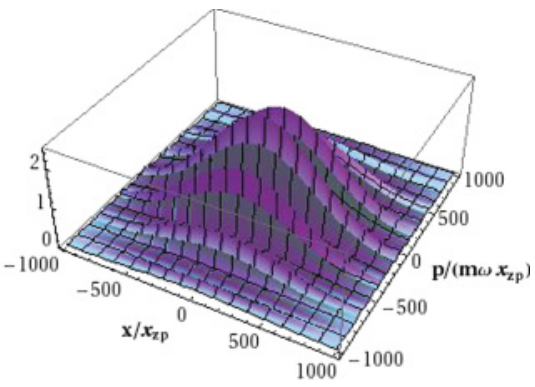

(c) $A=A_{0}$

FIG. 2. (Color online) Wigner distribution function of the membrane after one measurement giving the result $F_{y}=1$ and for several values of the backaction parameter: (a) $A=0.01 A_{0}$, (b) $A=0.1 A_{0}$, and (c) $A=A_{0}$, with $A_{0}=0.22 \sqrt{\hbar /\left(2 m \omega_{m}\right)}$. The evolution time is $t=\pi / \omega_{m}$ in all three cases. The Wigner function has been multiplied by $10^{6}$ to make the axes legible.

leads to the oscillations. We remark that the oscillations in the Wigner function can be seen as long as

$$
\frac{A}{x_{z p}} \gtrsim \sqrt{\tanh \eta}
$$

where $x_{z p}=\sqrt{\hbar /\left(2 m \omega_{m}\right)}$

\section{Backaction}

To illustrate the effect of backaction, we assume the following membrane parameters for the remainder of the paper (unless stated otherwise): $\omega_{m}=2 \pi \times 10^{6} \mathrm{rad} / \mathrm{s}, m=5 \times$ $10^{-13} \mathrm{~kg}, \mu_{m}=2 \times 10^{-11} \mathrm{~A} \mathrm{~m}^{2}$, and an initial temperature of $4 \mathrm{~K}$. The static external magnetic field is $B_{0}=0.1 \mathrm{G}$, and the condensate is $x_{0}=5 \times 10^{-6} \mathrm{~m}$ away from the membrane, resulting in a single-atom backaction parameter $A_{\mathrm{sa}}=9 \times$ $10^{-21} \mathrm{~m}$. We assume that $N=10^{5}$ atoms experience the same magnetic field in the detection region, yielding then an effective backaction parameter of $A_{0}=N A_{\mathrm{sa}}=9 \times 10^{-16} \mathrm{~m}$ (for comparison, $A_{0}=0.22 x_{z p}$ ). Figure 2 shows the resulting postmeasurement Wigner function for this specific value of $A_{0}$ [Fig. 2(c)] and for backaction parameters of $0.1 A_{0}$ [Fig. 2(b)] and $0.01 A_{0}$ [Fig. 2(a)], assuming that a measurement of $F_{y}$ with result 1 was made after an interaction time of $t_{1}=\pi / \omega_{m}$.

As alluded to by Eq. (18), in order to observe the oscillations in the postmeasurement Wigner function, we can either increase the temperature or increase $A$. However, increasing the temperature leads to dissipation and decoherence losses that are ignored in the present analysis but result, of course, in a fast thermalization and associated smoothing of $W(x, p)$. As mentioned above, these effects will be discussed in future work. A more promising approach to observe quantum interference effects is to increase $A$ [Eq. (9)], either by increasing the number of atoms in the effective detection zone or by increasing $B_{v}^{\prime}$ via a decrease of $x_{0}$. Since $B_{v}^{\prime}$ scales as $1 / x_{0}^{4}$, this may be the easiest way to reach the regime of observable Wigner function oscillations. Note, however, that for decreasing $x_{0}$, the simplified interaction Hamiltonian (6) becomes less accurate as the components of the magnetic field along $x$ and $y$ become more important, so the approximations made in Eq. (8) will not be as valid.
The expectation value of the center-of-mass position $\langle x\rangle$ of a membrane in thermal equilibrium is zero, and its variance is

$$
\sigma^{2}(x)=\left\langle x^{2}\right\rangle-\langle x\rangle^{2}=\left\langle x^{2}\right\rangle=\frac{\hbar}{2 m \omega_{m}} \operatorname{coth} \eta .
$$

Immediately following the measurement, the membrane is no longer in thermal equilibrium, and, in general, $\langle x\rangle \neq 0$. For large backaction parameters, the oscillations in $W(x, p)$ become quite significant. In that regime the BEC is a poor position sensor, since its coupling to the membrane significantly perturbs the outcome of subsequent measurements (see Fig. 2). Here, the measurement creates a significant change in the phase-space distribution of the membrane that invalidates any information gained about the position. However, as we show in Sec. $\mathrm{V}$, a high value of $A$ is beneficial for preparing the membrane in highly nonclassical states via repeated measurements. As with all schemes for state preparation involving repeated measurements, it is not very efficient for highly excited initial states, as is the case in Fig. 2. At $4 \mathrm{~K}$, and for the membrane parameters of this example, the mean phonon occupation number is $8.3 \times 10^{4}$.

To investigate the efficiency of our setup as a position sensor, it is therefore appropriate to consider the limit of small $A_{0}$. Consider, for concreteness, the specific example where the outcome of the spin measurement is $F_{y}=1$. Ignoring then terms of order $A^{2}$, and for $t_{1}=\pi / \Omega_{L 0}$, we find

$$
\langle x\rangle=-\frac{A}{2} \sin \left(\omega_{m} \pi / \Omega_{L 0}\right) \operatorname{coth} \eta,
$$

with $\left\langle x^{2}\right\rangle$ remaining constant to lowest order in $A$. We then have

$$
\sigma^{2}(x)_{\mathrm{pm}}=\frac{\hbar}{2 m \omega_{m}} \operatorname{coth} \eta-\frac{A^{2}}{4} \sin ^{2}\left(\omega_{m} \pi / \Omega_{L 0}\right) \operatorname{coth}^{2} \eta,
$$

where the subscript "pm" indicates "postmeasurement." The minimum backaction occurs for $\eta \rightarrow \infty$ or $T \rightarrow 0$. It also vanishes when the membrane frequency is an integer multiple of the Larmor precession frequency:

$$
\omega_{m}=n \Omega_{L 0},
$$

where $n$ is an integer, in which case it is possible to carry out stroboscopic quantum nondemolition (QND) measurements of the membrane position. 


\section{SUCCESSIVE MEASUREMENTS}

Generally, successive measurements of the condensate's spin result in an accumulation of backaction effects in the membrane. At the same time, they offer the potential for the coherent control of the center-of-mass motion of the membrane. To address such situations, we now consider the effect of a succession of measurements on the state of the membrane. The similarity between Eqs. (12) and (13) suggests that it should be possible to find a closed form for the density matrix of the composite system after an arbitrary number of measurements on the BEC. Indeed, this is the case and such a form is presented below.

\section{A. Analytical results}

We first consider the situation where the membrane begins in a thermal state and assume that the interaction between the membrane and the BEC (initially prepared in the $F_{x}=1$ state), is turned on at time $t_{0}=0$. After time $t_{1}$, a first measurement is performed on the spin of the BEC. The probe-BEC interaction time $t_{\text {meas }}$ required to carry out that measurement is taken to be negligible compared to the other characteristic times of the system-in practice this is true for phase contrast imaging. The direct effect of the measurement is only on the BEC and can be formally described by a Krauss operator $M^{(1)}$ that depends explicitly on the outcome of the measurement. Following that first measurement, the system evolves unitarily for an additional time $t_{2}$, becoming re-entangled. A second measurement is then performed, acting on the BEC with an operator $M^{(2)}$, and this process is repeated $n$ times. The set of operators $M^{\phi}$ are related to the above $W^{\phi}$ by $W^{\phi}=$ $M^{\phi \dagger} M^{\phi}$.

Immediately following the $n$th measurement, the elements of the BEC-membrane system's density matrix are given by (see Appendix B for more details)

$$
\begin{aligned}
\left\langle\alpha, x_{f}|\rho(t)| \beta, x_{i}\right\rangle= & \sum_{\alpha_{1}, \ldots \alpha_{n} ; \beta_{1}, \ldots \beta_{n}} M_{\alpha \alpha_{n}}^{(n)} M_{\alpha_{n} \alpha_{n-1}}^{(n-1)} \cdots M_{\alpha_{2} \alpha_{1}}^{(1)} \rho_{\alpha_{1} \beta_{1}} M_{\beta_{1} \beta_{2}}^{(1) \dagger} \cdots M_{\beta_{n-1} \beta_{n}}^{(n-1) \dagger} M_{\beta_{n} \beta}^{(n) \dagger} \\
& \times \exp \left\{-i \sum_{i=1}^{n} t_{i}\left[\Omega_{L 0}\left(\alpha_{i}-\beta_{i}\right)-\delta \Omega_{L}\left(\alpha_{i}^{2}-\beta_{i}^{2}\right)\right]\right\} \sqrt{\frac{m \omega_{m}}{\pi \hbar} \tanh \eta} \\
& \times \exp \left(-\frac{m \omega_{m}}{4 \hbar}\left\{\left[x_{f}+X(\alpha) A+x_{i}+X(\beta) A\right]^{2} \tanh \eta+\left[x_{f}+X(\alpha) A-x_{i}-X(\beta) A\right]^{2} \operatorname{coth} \eta\right.\right. \\
& \left.\left.+4 i A\left(P(\alpha)\left[x_{f}+X(\alpha) A\right]-P(\beta)\left[x_{i}+X(\beta) A\right]\right)+2 i A^{2}[\phi(\alpha)-\phi(\beta)]\right\}\right),
\end{aligned}
$$

where

$$
\begin{gathered}
M_{\sigma \tau}^{(i)}=\left\langle\sigma\left|M^{(i)}\right| \tau\right\rangle \\
X(\sigma)=\sigma_{n}-\sum_{i=1}^{n}\left(\sigma_{i}-\sigma_{i-1}\right) \cos T_{i, n} \\
P(\sigma)=\sum_{i=1}^{n}\left(\sigma_{i}-\sigma_{i-1}\right) \sin T_{i, n} \\
\phi(\sigma)=\sum_{i=1}^{n}\left(\sigma_{i}-\sigma_{i-1}\right)^{2} \sin T_{i, n} \cos T_{i, n} \\
+2 \sum_{i=1}^{n-1} \sum_{j=i+1}^{n}\left(\sigma_{i}-\sigma_{i-1}\right)\left(\sigma_{j}-\sigma_{j-1}\right) \sin T_{j, n} \cos T_{i, n} \\
T_{i, j}= \begin{cases}\omega_{m} \sum_{k=i}^{j} t_{k} & \text { if } \quad i \leqslant j \\
0 & \text { if } \quad i>j\end{cases}
\end{gathered}
$$

The sums on $\alpha_{i}$ and $\beta_{i}$ run over $\{-1,0,+1\}$ and, in Eqs. (25)-(27), we have $\alpha_{0}=\beta_{0}=0$ wherever they appear. To be clear, in the equations above, the quantities $t_{i}$ are the intervals between measurements rather than the times themselves. Also, care should be taken because $X, P$, and $\phi$ are functions of the spin indices and thus must be recomputed for each term in the sum. Note also that the density matrix (23) is unnormalized. Its trace is equal to the probability of the particular sequence of measurement outcomes described by the specific set of operators $\left\{M^{(i)}\right\}$ that occur in it.

A few remarks are in order before turning to a discussion of numerical results: First, the density matrix of the membrane can be obtained from Eq. (23) by a partial trace. It is the sum of many different contributions from various shifted (in position and momentum) thermal ensembles. A (numerical) computation problem does arise, however, because calculating the density matrix for $n$ measurements requires summing over $9^{n}$ terms. In our numerics we are thus restricted to few-measurement scenarios. The timing of the measurements is also very important to the BEC functioning as a detector. In one extreme case, if the measurements are made exactly at the natural frequency of the membrane, the interaction would be completely masked, except for the tiny second-order effect of $\delta \Omega_{L}$. This supports the claim that position is very nearly a stroboscopic QND variable.

The structures of Eqs. (23) and (25)-(27) indicate that the results of the earliest measurements continue to be as important as those of later measurements. This is because, in the absence of dissipation, there is no attenuation of the information gained nor of the backaction induced by the measurements. Including the effects of thermal dissipation reduces and eventually erases this memory effect. 
The Wigner function (15) of the membrane after $n$ measurements can be derived from (23) and is found to be

$$
\begin{aligned}
W(x, p)= & \frac{1}{\operatorname{tr}\left(\rho_{\text {sys }}(t)\right)} \sum_{\gamma, \alpha_{1}, \ldots, \beta_{n}} M_{\gamma \alpha_{n}}^{(n)} M_{\alpha_{n} \alpha_{n-1}}^{(n-1)} \cdots M_{\alpha_{2} \alpha_{1}}^{(1)} \rho_{\alpha_{1} \beta_{1}} M_{\beta_{1} \beta_{2}}^{(1) \dagger} \cdots M_{\beta_{n-1} \beta_{n}}^{(n-1) \dagger} M_{\beta_{n} \gamma}^{(n) \dagger} \\
& \times \exp \left\{-i \sum_{i=1}^{n} t_{i}\left[\Omega_{L 0}\left(\alpha_{i}-\beta_{i}\right)-\delta \Omega_{L}\left(\alpha_{i}^{2}-\beta_{i}^{2}\right)\right]\right\} \frac{1}{\pi \hbar} \tanh \eta \\
& \times \exp \left\{-\frac{m \omega_{m}}{\hbar}\left[\left(\left\{x+\frac{A}{2}[X(\alpha)+X(\beta)]\right\}^{2}+\left\{\frac{p}{m \omega_{m}}+\frac{A}{2}[P(\alpha)+P(\beta)]\right\}^{2}\right) \tanh \eta\right.\right. \\
& \left.\left.+i A[P(\alpha)-P(\beta)]\left\{x+\frac{A}{2}[X(\alpha)+X(\beta)]\right\}-i A[X(\alpha)-X(\beta)] \frac{p}{m \omega_{m}}+i \frac{A^{2}}{2}[\phi(\alpha)-\phi(\beta)]\right]\right\}
\end{aligned}
$$

a form that clearly illustrates the shift in position and momentum of the various terms in the sum.

\section{B. Numerical results}

One simple way to detect the effect of quantum backaction on the state of the membrane is to consider a sequence of 2 measurements carried out in succession at times $t_{1}$ and $t_{2}$ and to compare the outcome of the last measurement to the outcome of a measurement at that same time $t_{2}$ but skipping the first measurement at $t_{1}$. As a concrete example, we consider, for the parameters of the previous section, the following two scenarios:

(1) $F_{y}$ is measured once, at $t_{2}=\pi / \omega_{m}$ (i.e., at half the oscillator period),

(2) $F_{y}$ is measured first at $t_{1}=\pi /\left(2 \omega_{m}\right)$ and then at $t_{2}=$ $\pi / \omega_{m}$.

By simply calculating the trace of Eq. (23) for the three possible operators $M^{(1)}$ in scenario 1 we obtain the probabilities

$$
\begin{gathered}
P\left(F_{y}= \pm 1\right)=0.375, \\
P\left(F_{y}=0\right)=0.25 .
\end{gathered}
$$

In the second scenario, nine traces need to be evaluated instead of just three. Summing then the three terms corresponding to the same final value of $F_{y}$ so as to obtain the total probability of obtaining a particular value for the second measurement, we find

$$
\begin{gathered}
P\left(F_{y}= \pm 1\right)=0.344, \\
P\left(F_{y}=0\right)=0.312 .
\end{gathered}
$$

This simple example shows that backaction of the intermediate measurement should be readily observable, since it changes the probability for the three possible outcomes of the measurements at $t_{2}$ by a significant amount; of the order of several percent.

A slightly more complete look into the effects of intermediate measurements is provided by the Wigner functions shown in Figs. 2 and 3. In Figs. 2(a)-2(c), we show a series of plots for the case of a single measurement at $t=\pi / \omega_{m}$ for different values of $A\left(0.01 A_{0}, 0.1 A_{0}\right.$, and $A_{0}$, respectively). In Fig. 3 we increase the number of measurements from 1 to
2. The time of the final measurement is the same as in Fig. 2 (i.e., $\left.\pi / \omega_{m}\right)$, but an intermediate measurement is made at time $t_{1}=\pi /\left(2 \omega_{m}\right)$. The outcome of the final spin measurement is again $F_{y}=1$ but, for the sake of direct comparison with Fig. 2, we average over all possible results of the intermediate measurement. This is equivalent to an experiment in which the outcomes of the intermediate measurement are discarded or ignored. As expected, the Wigner functions with an intermediate measurement are noticeably different than those lacking an intermediate measurement, hinting at a simple way to characterize the impact of quantum backaction on the membrane dynamics.

\section{INITIAL COHERENT STATE}

We now turn to the situation where the center-of-mass state of the membrane is a coherent state, which is an initial condition that can be prepared by driving the membrane with a classical force. The main result of this section is to demonstrate that, after $n$ measurements, the center of mass of the membrane is split into a superposition of up to $3^{n}$ discrete coherent states, hinting at the possibility of generating a macroscopic Schrödinger cat.

\section{A. Evolution of the coherent state}

The assumption that the membrane is initially in a coherent state allows us to eschew the density matrix formalism for the moment, and the initial state of the composite system is given by

$$
|\Psi(0)\rangle=D\left(a_{0}+i b_{0}\right)\left|0_{\mathrm{mem}}\right\rangle \otimes \sum_{\alpha} c_{\alpha}|\alpha\rangle,
$$

where $\sum_{\alpha}\left|c_{\alpha}\right|^{2}=1$ and

$$
D(u)=\exp \left(u \hat{a}^{\dagger}-u^{*} \hat{a}\right)
$$

is the displacement operator for the center-of-mass state of the membrane. Following a procedure similar to that for the 


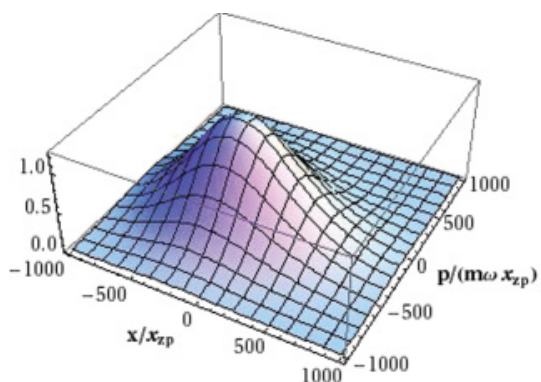

(a) $A=0.01 A_{0}$

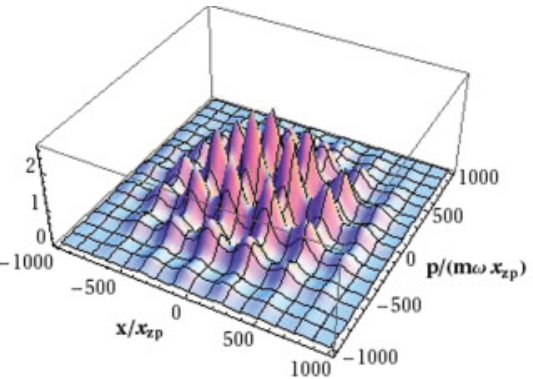

(b) $A=0.1 A_{0}$

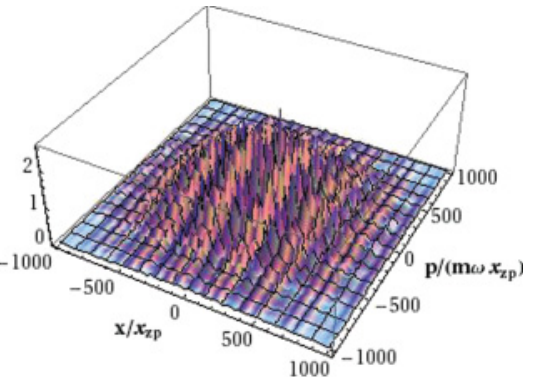

(c) $A=A_{0}$

FIG. 3. (Color online) Wigner distribution function of the membrane in the case of two measurements: the final spin measurement, at time $\pi / \omega_{m}$, yields the outcome $F_{y}=1$. As in Fig. (2), the three Wigner functions are plotted for increasing values of the backaction parameter $A$ and the final Wigner functions are averaged over all possible outcomes of the intermediate measurement.

initially thermal membrane, we arrive at the final state for the hybrid membrane-BEC system (see Appendix C):

$$
\begin{aligned}
|\Psi(0)\rangle= & \sum_{\alpha, \alpha_{1}, \ldots, \alpha_{n}} M_{\alpha \alpha_{n}}^{(n)} \cdots M_{\alpha_{2} \alpha_{1}}^{(1)} c_{\alpha_{1}} \\
& \times \exp \left[-i \sum_{i=1}^{n} t_{i}\left(\Omega_{L 0} \alpha_{i}-\delta \Omega_{L} \alpha_{i}^{2}+\frac{\omega_{m}}{2}\right)\right. \\
& \left.-i \frac{A \Theta(\alpha)}{2 x_{z p}}\right] D[a(\alpha)+i b(\alpha)]\left|0_{\mathrm{mem}}\right\rangle \otimes|\alpha\rangle,
\end{aligned}
$$

with

$$
\begin{aligned}
a(\sigma)= & a_{0} \cos T_{1, n}+b_{0} \sin T_{1, n}-\frac{A}{2 x_{z p}} X(\sigma), \\
b(\sigma) & =b_{0} \cos T_{1, n}-a_{0} \sin T_{1, n}-\frac{A}{2 x_{z p}} P(\sigma), \\
\Theta(\sigma)= & \sum_{i=1}^{n} \sigma_{i}\left[a_{0}\left(\sin T_{1, i}-\sin T_{1, i-1}\right)-b_{0}\left(\cos T_{1, i}\right.\right. \\
& \left.-\cos T_{1, i-1}\right)+\frac{A}{2 x_{z p}} \sum_{j=1}^{i}\left(\sigma_{j}-\sigma_{j-1}\right) \\
& \left.\times\left(\sin T_{j, i}-\sin T_{j, i-1}\right)\right]
\end{aligned}
$$

and all other definitions are as before. This wave function is not normalized; the probability of obtaining a particular sequence of measurement outcomes is given by $\langle\Psi(t) \mid \Psi(t)\rangle$.

Because the real part of the displacement is proportional to $\langle x\rangle$ and the imaginary part is proportional to $\langle p\rangle$, we have a very clear picture of the physics arising in this situation. The membrane's initial displacement oscillates back and forth semiclassically. However, each time the BEC's spin is measured, the membrane's wave function splits into three distinct components, each having received a different kick from its interaction with the different possible spin orientations of the BEC. This would indicate that the membrane was indeed put into a Schrödinger-cat state, except for the fact that the kick is proportional to $A$, which is typically at most comparable in size to the zero-point oscillations $x_{z p}$. If, going forward, we can increase $A$ in an experimentally realizable setting, this system may provide an excellent demonstration of a macroscopic object put into a highly nonclassical state.

\section{B. Wigner function}

Following a sequence of measurements, the Wigner function of the initially coherent state of the membrane motion becomes

$$
\begin{aligned}
W(x, p)= & \frac{1}{\pi \hbar\langle\Psi(t) \mid \Psi(t)\rangle} \sum_{\gamma, \alpha_{1}, \ldots, \beta_{n}} M_{\gamma \alpha_{n}}^{(n)} M_{\alpha_{n} \alpha_{n-1}}^{(n-1)} \cdots M_{\alpha_{2} \alpha_{1}}^{(1)} \rho_{\alpha_{1} \beta_{1}} M_{\beta_{1} \beta_{2}}^{(1) \dagger} \cdots M_{\beta_{n-1} \beta_{n}}^{(n-1) \dagger} M_{\beta_{n} \gamma}^{(n) \dagger} \\
& \times \exp \left\{-i \sum_{i=1}^{n} t_{i}\left[\Omega_{L 0}\left(\alpha_{i}-\beta_{i}\right)-\delta \Omega_{L}\left(\alpha_{i}^{2}-\beta_{i}^{2}\right)\right]\right\} \\
& \times \exp \left\{-\frac{m \omega_{m}}{\hbar}\left[\left(x-x_{z p}\{a(\alpha)+a(\beta)+i[b(\alpha)-b(\beta)]\}\right)^{2}\right.\right. \\
& \left.+\left(\frac{p}{m \omega_{m}}-x_{z p}\{b(\alpha)+b(\beta)-i[a(\alpha)-a(\beta)]\}\right)^{2}\right]+i[a(\beta) b(\alpha)-a(\alpha) b(\beta)] \\
& \left.-i \frac{A}{2 x_{z p}}[\Theta(\alpha)-\Theta(\beta)]-\frac{1}{2}[a(\alpha)-a(\beta)]^{2}-\frac{1}{2}[b(\alpha)-b(\beta)]^{2}\right\} .
\end{aligned}
$$


Figure 4 shows the postmeasurement Wigner function after successive measurements. The key point here is that repeated measurements can lead to very nonclassical states, as is evident from the resulting negative valued Wigner functions. As can be seen in Eqs. (34) and (38), each measurement introduces different phase factors to the initial coherent state, along with splitting it into different coherent states. It is the quantum interference between the different coherent states thus generated that gives rise to the nonclassical Wigner functions. Relatively few measurements are required to generate such nonclassical states. In fact, it is possible to turn a coherent state into a nonclassical state after only one measurement. Figure 4 shows one such case. Here, $a_{0}=b_{0}=1$ and the result of the first measurement after a time interval of $t_{1}=\pi / \omega_{m}$ is $F_{y}=0$. Repeated measurements after equal time intervals lead to states that resemble displaced Fock states and other more complex states.

If the initial displacement is small in magnitude (as in Fig. 4), production of this nonclassical behavior is rather insensitive to the measurement timing. For this particular case, with the outcome $F_{y}=0$, it is seen for $t_{1}$ in the approximate range $0.6 \pi / \omega_{m}-1.8 \pi / \omega_{m}$. On the other hand, if the initial displacement is large (for example, $a_{0}=50 \sqrt{2}, b_{0}=0$ ), the nonpositive Wigner function is much harder to find; in this case it only occurs from $t_{1} \approx 0.77 \pi / \omega_{m}$ to $0.79 \pi / \omega_{m}$. Nevertheless, in either case, successive measurements can build up some very interesting nonclassical states. It may be possible to obtain a high degree of probabilistic quantum-state control of the membrane if the dissipative effects do not wash out the interference too quickly.

\section{CONCLUSION}

In conclusion, we have demonstrated that, by coupling a magnetic membrane to a spinor BEC, we can monitor and manipulate the position of the membrane. A projective measurement procedure induces significant backaction that can be measured for reasonable experimental parameters. We investigated the effect of this interaction for different initial membrane states; namely, thermal and coherent states. We discussed the possibility to measure backaction of a quantum measurement on the membrane via repeated measurements and the potential to generate Schrödinger-cat states of the oscillator via such repeated measurements in the case of an initial coherent state. It would be a major accomplishment to prepare such a state of a solid, macroscopic object.

As mentioned above, we will look in future work at the effects of using a more dispersive (and more experimentally plausible) measurement scheme based on observation of the Larmor precession. Additionally, we will study the effects of including dissipation on the system's dynamics. Other possibilities for extending the model even further include inclusion of a general coupling to other spin components (i.e., an interaction Hamiltonian of the form $\mathbf{F} \cdot \mathbf{B}$ ) because, at short

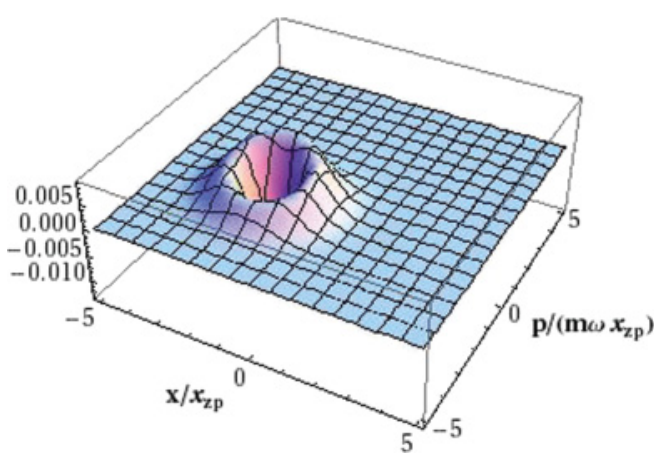

(a) 1st meas. $F_{y}=0, t_{1}=\pi / \omega_{m}$

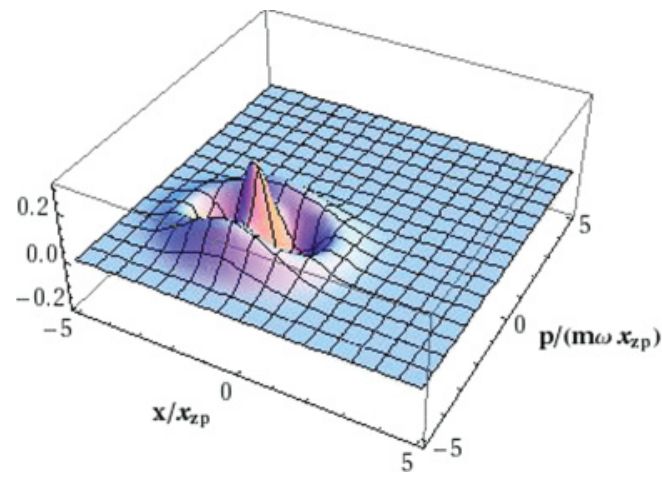

(c) 3rd meas. $F_{y}=-1, t_{3}=\pi / \omega_{m}$

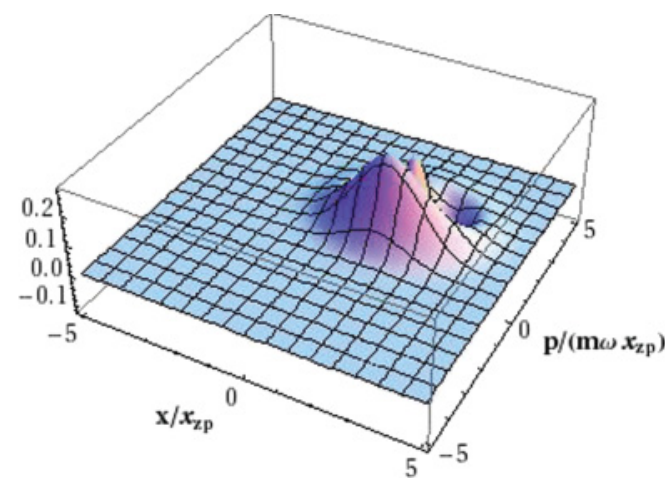

(b) 2nd meas. $F_{y}=1, t_{2}=\pi / \omega_{m}$

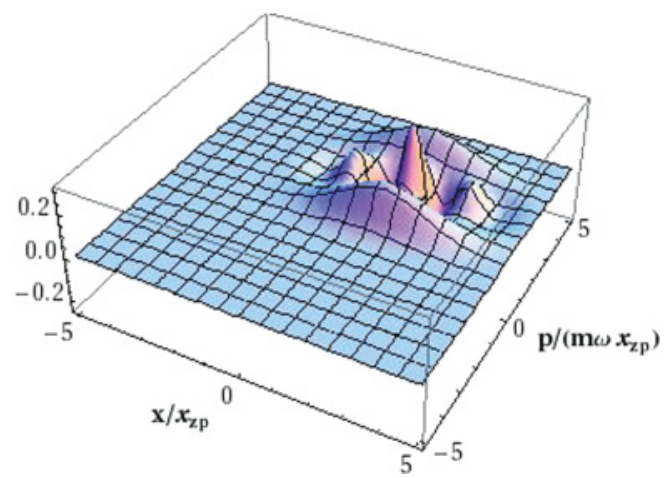

(d) 4th meas. $F_{y}=1, t_{4}=\pi / \omega_{m}$

FIG. 4. (Color online) Postmeasurement Wigner distribution functions of the membrane, initially in a coherent state $\alpha=1+i 1$. We notice that repeated measurements do indeed lead to some interesting nonclassical states. The measurement results and evolution time before measurement are given for each plot. 
distances, the $x$ and $y$ gradients of the magnetic field become significant. Also, the BEC is a spatially finite system, so we may wish to exploit the ability to measure multiple "pixels" of the condensate in order to gain better information about the membrane's position and/or better control of the membrane's state.

\section{ACKNOWLEDGMENTS}

We thank David Brown for his help during the initial stages of these calculations. This work was supported by the DARPA QuASAR program through a Grant from AFOSR and the DARPA ORCHID program through a Grant from ARO, the US Army Research Office, and the NSF. M.V. acknowledges support from the Alfred P. Sloan Foundation. MET is supported by TUBITAK (the Scientific and Technological Research Council of Turkey).

\section{APPENDIX A: DERIVATION OF INTERACTION HAMILTONIAN}

Our starting assumption is that the magnetic domain on the membrane is a point dipole located at the origin.

The magnetic field due to that dipole is

$$
\mathbf{B}(\mathbf{r})=\frac{\mu_{0}}{4 \pi} \frac{1}{r^{3}}\left[3\left(\boldsymbol{\mu}_{m} \cdot \hat{\mathbf{r}}\right) \hat{\mathbf{r}}-\boldsymbol{\mu}_{m}\right] .
$$

It is the small inhomogeneity of the resulting magnetic field along the long axis $z$ of the condensate, and the resulting variation in Larmor precession frequency, that permits us to characterize the center-of-mass mode of oscillation of the membrane.

For a dipole polarized along the $z$ axis $\left(\boldsymbol{\mu}_{m}=\mu_{m} \hat{\mathbf{z}}\right)$, at distance $r=\left(x^{2}+y^{2}+z^{2}\right)^{1 / 2}$, the components of the magnetic field (A1) are

$$
\begin{gathered}
B_{x}(x, y, z)=\frac{\mu_{0}}{4 \pi} \frac{\mu_{m}}{r^{3}}\left[\frac{3 x z}{r^{2}}\right], \\
B_{y}(x, y, z)=\frac{\mu_{0}}{4 \pi} \frac{\mu_{m}}{r^{3}}\left[\frac{3 y z}{r^{2}}\right], \\
B_{z}(x, y, z)=\frac{\mu_{0}}{4 \pi} \frac{\mu_{m}}{r^{3}}\left[\frac{3 z^{2}}{r^{2}}-1\right] .
\end{gathered}
$$

Let $x=x_{0}+x_{m}$, where $x_{0}$ is the equilibrium value of $x$ for a condensate atom, $x=0$ is the equilibrium position of the membrane, and $x_{m}$ is the small sinusoidal displacement of the membrane around the origin. Expanding the expression for magnetic fields for small $x_{m}$ (up to first order), we get

$$
\begin{gathered}
B_{x} \approx \frac{\mu_{0} \mu_{m}}{4 \pi r_{0}^{5}}\left[3 x_{0} z-\frac{3 z\left(4 x_{0}^{2}-y^{2}-z^{2}\right)}{r_{0}^{2}} x_{m}\right], \\
B_{y} \approx \frac{\mu_{0} \mu_{m}}{4 \pi r_{0}^{5}}\left[3 y z-\frac{15 x_{0} y z}{r_{0}^{2}} x_{m}\right], \\
B_{z} \approx \frac{\mu_{0} \mu_{m}}{4 \pi r_{0}^{5}}\left[\left(2 z^{2}-x_{0}^{2}-y^{2}\right)+\frac{3 x_{0}\left(x_{0}^{2}+y^{2}-4 z^{2}\right)}{r_{0}^{2}} x_{m}\right] .
\end{gathered}
$$

Here, $r_{0}=\left(x_{0}^{2}+y^{2}+z^{2}\right)^{1 / 2}$. Assuming a two-dimensional condensate (i.e., $y \approx 0$ ), we can set $B_{y} \rightarrow 0$. For theoretical simplicity, we assume that the measurements are made on the part of the condensate directly above the magnet (i.e., close to $z=0$ ). Under these approximations, the magnetic field simplifies to being predominantly in the $z$ direction:

$$
B_{z} \approx \frac{\mu_{0} \mu_{m}}{4 \pi x_{0}^{4}}\left[-x_{0}+3 x_{m}\right] .
$$

Combining this with the quantization field $B_{0}$, we end up with the interaction Hamiltonian of the form

$$
V=\mu_{B} g_{F} F_{z}\left(B_{0}-\frac{\mu_{0} \mu_{m}}{4 \pi x_{0}^{3}}+\frac{3 \mu_{0} \mu_{m}}{4 \pi x_{0}^{4}} x_{m}\right) .
$$

We can break this total magnetic field into a constant part $B_{c}$ (the sum of the first two terms) and a component that varies at the membrane frequency, $B_{v}^{\prime} x_{m}$. Here, the gradient of the magnetic field is

$$
B_{v}^{\prime}=\frac{3 \mu_{0} \mu_{m}}{4 \pi x_{0}^{4}} .
$$

This is the form used in Eq. (4).

\section{APPENDIX B: DERIVATION OF SUCCESSIVE MEASUREMENT DENSITY MATRIX}

Here we outline the steps used in Sec. IV to derive Eq. (23). We arrive at the result via induction. Suppose that $n-1$ measurements have been performed already. We denote the density matrix at this moment by $\rho^{(n-1)}$. After the next period of free evolution and the $n$th measurement, we have

$$
\rho^{(n)}=M^{(n)} U\left(t_{n}\right) \rho^{(n-1)} U^{\dagger}\left(t_{n}\right) M^{(n) \dagger} .
$$

To find the matrix elements, we insert completeness relationships

$$
\begin{aligned}
& \left\langle\alpha, x_{f}\left|\rho^{(n)}\right| \beta, x_{i}\right\rangle \\
& =\sum_{\alpha_{n}} \sum_{\beta_{n}} \int d x \int d y\left\langle\alpha, x_{f}\left|M^{(n)} U\left(t_{n}\right)\right| \alpha_{n}, x\right\rangle \\
& \quad \times\left\langle\alpha_{n}, x\left|\rho^{(n-1)}\right| \beta_{n}, y\right\rangle\left\langle\beta_{n}, y\left|U^{\dagger}\left(t_{n}\right) M^{(n) \dagger}\right| \beta, x_{f}\right\rangle .
\end{aligned}
$$

Now, the propagator can be derived from Eq. (12) by multiplying by the trace, substituting $\eta \rightarrow i \omega_{m} t / 2$, and including the contributions of the $F_{z}$-dependent parts of the Hamiltonian. Using these techniques, we arrive at the needed matrix elements:

$$
\begin{aligned}
& \left\langle\sigma, u\left|M^{(n)} U\left(t_{n}\right)\right| \tau, v\right\rangle \\
& =M_{\sigma \tau}^{(n)} \sqrt{\frac{8 m \omega_{m}}{i \pi \hbar \sin \omega_{m} t}} \exp \left(-i\left\{t_{n} \Omega_{L 0} \tau-t_{n} \delta \Omega \tau^{2}\right.\right. \\
& \quad+\frac{m \omega}{4 \hbar}\left[(u+v+2 A \tau)^{2} \tan \frac{\omega_{m} t_{n}}{2}\right. \\
& \left.\left.\left.\quad-(u-v)^{2} \cot \frac{\omega_{m} t_{n}}{2}\right]\right\}\right) .
\end{aligned}
$$

The fact that Eq. (12) and the individual terms of Eq. (14) [i.e., terms of the form of Eq. (13)] do not differ, except for shifts in the coordinates and some complex phase shifts, leads us to conjecture that additional evolution and measurements will not alter the underlying structure of the density matrix. Thus, we try a matrix of the form (23) for $\rho^{(n-1)}$, but with 
unknowns for $X^{(n-1)}$, etc. (superscript added for clarity), and see if evaluation of the integral (B2) produces a similar output for $\rho^{(n)}$. Indeed it does, and it also gives us the recurrence relations used to derive Eqs. (25)-(27). These are

$$
\begin{aligned}
& X^{(n)}(\sigma)= X^{(n-1)}(\sigma) \cos \omega_{m} t_{n}+P^{(n-1)}(\sigma) \sin \omega_{m} t_{n} \\
&+\sigma_{n}\left(1-\cos \omega_{m} t_{n}\right) \\
& P^{(n)}(\sigma)= P^{(n-1)}(\sigma) \cos \omega_{m} t_{n}-X^{(n-1)}(\sigma) \sin \omega_{m} t_{n} \\
&+\sigma_{n} \sin \omega_{m} t_{n}, \\
& \phi^{(n)}(\sigma)=\phi^{(n-1)}(\sigma)+\left[X^{(n)}(\sigma)^{2}+X^{(n-1)}(\sigma)^{2}\right] \cot \omega_{m} t_{n} \\
&- 2 X^{(n)}(\sigma) X^{(n-1)}(\sigma) \csc \omega_{m} t_{n} .
\end{aligned}
$$

The solutions to these recurrence relationships are the equations given above.

\section{APPENDIX C: DERIVATION OF COHERENT-STATE EVOLUTION}

We again will arrive at the result (34) by using induction and finding a set of recurrence relations as in Appendix B, but the steps involved are slightly different. We first split the Hamiltonian (8) into two parts: $\tilde{H}_{\mathrm{BEC}}$ and $\left(\tilde{H}_{m}+\tilde{V}\right)$. These commute, so the unitary evolution operator can simply be factorized into two operators, one acting only on the BEC and another acting as a shifted harmonic oscillator. We also note that the unperturbed membrane Hamiltonian and $\tilde{H}_{m}+\tilde{V}$ are related by a simple unitary transformation; namely,

$$
\tilde{H}_{m}+\tilde{V}=D\left(-\frac{A}{2 x_{z p}} F_{z}\right) H_{m} D\left(\frac{A}{2 x_{z p}} F_{z}\right) .
$$

Using this fact and the well-known results

$$
\begin{gathered}
\exp \left(-i H_{m} t / \hbar\right) D(\zeta)=D\left(e^{-i \omega_{m} t} \zeta\right) \exp \left(-i H_{m} t / \hbar\right), \\
D(\zeta) D(\xi)=D(\zeta+\xi) e^{\left(\zeta \xi^{*}-\zeta^{*} \xi\right) / 2}
\end{gathered}
$$

we can postulate that the system after $n-1$ measurements is specified by a superposition of states, as in (34), apply an additional evolution and measurement,

$$
\left|\Psi^{(n)}\right\rangle=M^{(n)} \tilde{U}_{\mathrm{BEC}}\left(t_{n}\right) \tilde{U}_{m}\left(t_{n}\right)\left|\Psi^{(n-1)}\right\rangle,
$$

and then use the result to find the following recurrences:

$$
\begin{aligned}
& a^{(n)}(\sigma)= a^{(n-1)}(\sigma) \cos \omega_{m} t_{n}+b^{(n-1)}(\sigma) \sin \omega_{m} t_{n} \\
&-\frac{\sigma_{n} A}{2 x_{z p}}\left(1-\cos \omega_{m} t_{n}\right), \\
& b^{(n)}(\sigma)= b^{(n-1)}(\sigma) \cos \omega_{m} t_{n}-a^{(n-1)}(\sigma) \sin \omega_{m} t_{n} \\
&-\frac{\sigma_{n} A}{2 x_{z p}} \sin \omega_{m} t_{n}, \\
& \Theta^{(n)}=\Theta^{(n-1)}+\alpha_{n}\left(b^{(n-1)}-b^{(n)}\right) .
\end{aligned}
$$

Note the strong resemblance between the first two of these equations and those from the previous appendix. This is to be expected, because the real and imaginary parts of the displacement are proportional to the expected position and momentum of the coherent state, respectively. The solutions to these equations are Eqs. (35)-(37).

\section{APPENDIX D: EXPERIMENTAL IMPLEMENTATION}

Here, we provide details on the experimental detection of the quantum backaction on the micromechanical membrane. The membrane is composed of silicon nitride with a micron-scale magnetic domain deposited at its center. These structures are fabricated by coating silicon wafers with around $100 \mathrm{~nm}$ of high-stress silicon nitride and then etching the silicon in a $100 \mu \mathrm{m} \times 100 \mu \mathrm{m}$ region to reveal a free-standing membrane. Quality factors exceeding $10^{6}$ have been demonstrated with similar $\mathrm{SiN}$ membranes for resonance frequencies in the range of $500 \mathrm{kHz}$ [17].

The magnetic domain is deposited on this membrane by a combination of photolithography and sputtering of a high-permeability material like permalloy [18]. Peak surface fields on the order of $1 \mathrm{~T}$ at the surface of this magnetic domain should ensure field gradients around $10^{3} \mathrm{~T} / \mathrm{m}$ in the regions above the domain. The membrane is supported on a cryogenically cooled flange housed in an ultrahigh-vacuum (UHV) chamber. Sputtered gold films deposited on the substrate in the regions surrounding the membrane serve as mirrors for trapping and imaging. Spinor Bose condensates of ${ }^{87} \mathrm{Rb}$ in the $F=1$ hyperfine state will be optically confined about $5 \mu \mathrm{m}$ above the membrane in a quasi-two-dimensional (quasi-2D) trap created by shallow-angle interference of laser beams reflected off the gold surface [19]. This ensures a quasi-2D confinement of the condensates such that the spatial extent of the gas in the direction normal to the membrane is less than the spin healing length $\xi=\sqrt{\hbar^{2} /\left(2 m\left|c_{2} n\right|\right)}$. Here, $c_{2}=4 \pi \hbar^{2}\left(a_{2}-a_{0}\right) / m$ is the spin-dependent coupling strength of the spinor gas and $a_{0}\left(a_{2}\right)$ is the $s$-wave scattering length in the $F=0(F=2)$ channel. In this way, we can freeze out spin dynamics along this dimension [5].

The estimation of the membrane's position is based on detecting the Zeeman shift at the location of the condensate due to the micromotion of the membrane. This is achieved by magnetization-sensitive phase contrast imaging as demonstrated in [5]. To summarize this technique briefly, a sequence of nondestructive phase contrast images of the condensate are obtained with far-off-resonant circular polarized light. Due to Larmor precession of the gas, the phase imprinted on the probe light is modulated at the Larmor frequency leading to a phase contrast signal given by

$$
s=1+2 \tilde{n} \sigma_{0}(\gamma / 2 \Delta)\left[a_{0}+a_{1}\left\langle F_{y}\right\rangle+a_{2}\left\langle F_{y}^{2}\right\rangle\right],
$$

where $\tilde{n}$ is the column density of the gas, $\sigma_{0}=3 \lambda^{2} /(2 \pi)$ is the resonant cross section, $\Delta$ is the detuning of the probe light from resonance and $\gamma$ is the natural linewidth. $F_{y}$ is the projection of the local atomic spin along the imaging axis. The constants $a_{0}, a_{1}$, and $a_{2}$ depend on the detuning of the probe light and describe the isotropic polarization and optical activity of the condensate. By analyzing the sequence of phase contrast images on a pixel-by-pixel basis, we can estimate the local Larmor precession rate and, thus, the local magnetic field. At the shot-noise limit, we estimate a magnetic field sensitivity on the order of $15 \mathrm{fT} / \mathrm{Hz}^{1 / 2}$ for a 
condensate of area $50 \mu \mathrm{m}^{2}$ in the plane of the membrane [20]. For the expected field gradient of $10^{3} \mathrm{~T} / \mathrm{m}$ at the location of the condensate, this field sensitivity translates to a position sensitivity of $15 \times 10^{-18} \mathrm{~m} / \mathrm{Hz}^{1 / 2}$. This is much smaller than the zero-point motion of the oscillator which has an amplitude on the order of $6 \times 10^{-15} \mathrm{~m}$.
[1] V. B. Braginsky and F. Ya. Khalili, Quantum Measurement (Cambridge University Press, Cambridge, 1992).

[2] T. J. Kippenberg and K. J. Vahala, Science 321, 1172 (2008); F. Marquardt and S. M. Girvin, Physics 2, 40 (2009).

[3] Alain Aspect, Jean Dalibard, and Gérard Roger, Phys. Rev. Lett. 49, 1804 (1982).

[4] James N. Hollenhorst, Phys. Rev. D 19, 1669 (1979).

[5] M. Vengalatorre, J. M. Higbie, S. R. Leslie, J. Guzman, L. E. Sadler, and D. M. Stamper-Kurn, Phys. Rev. Lett. 98, 200801 (2007).

[6] J. M. Obrecht, R. J. Wild, M. Antezza, L. P. Pitaevskii, S. Stringari, and E. A. Cornell, Phys. Rev. Lett. 98, 063201 (2007).

[7] T. van Zoest, Science 328, 1540 (2010).

[8] J. M. Higbie, L. E. Sadler, S. Inouye, A. P. Chikkatur, S. R. Leslie, K. L. Moore, V. Savalli, and D. M. Stamper-Kurn, Phys. Rev. Lett. 95, 050401 (2005).

[9] Ying-Ju Wang, M. Eardley, S. Knappe, J. Moreland, L. Hollberg, and J. Kitching, Phys. Rev. Lett. 97, 227602 (2006).
[10] D. Hunger, S. Camerer, T. W. Hansch, D. Konig, J. P. Kotthaus, J. Reichel, and P. Treutlein, Phys. Rev. Lett. 104, 143002 (2010)

[11] P. Treutlein, D. Hunger, S. Camerer, T. W. Hansch, and J. Reichel, Phys. Rev. Lett. 99, 140403 (2007).

[12] C. Genes, D. Vitali, and P. Tombesi, Phys. Rev. A 77, 050307 (2008).

[13] K. Hammerer, M. Wallquist, C. Genes, M. Ludwig, F. Marquardt, P. Treutlein, P. Zoller, J. Ye, and H. J. Kimble, Phys. Rev. Lett. 103, 063005 (2009).

[14] S. Singh and P. Meystre, Phys. Rev. A 81, 041804 (2010).

[15] K. Hammerer, K. Stannigel, C. Genes, P. Zoller, P. Treutlein, S. Camerer, D. Hunger, and T. W. Hansch, Phys. Rev. A 82, 021803 (2010).

[16] K. W. Murch, Nature Phys. 4, 561 (2008).

[17] B. M. Zwickl et al., Appl. Phys. Lett. 92, 103125 (2008).

[18] M. Vengalattore et al., J. Appl. Phys. 95, 4404 (2004).

[19] D. Gallego et al., Opt. Lett. 34, 3463 (2009).

[20] L. M. Aycock, S. Chakram, and M. Vengalattore (to be published). 